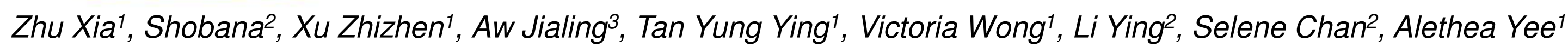
${ }^{1}$ Division of Supportive and Palliative Care, National Cancer Centre Singapore; ${ }^{2}$ Nursing Department, Singapore General Hospital;

\title{
Achieving timely symptom control for patients needing opioid infusions: A quality improvement project
}

\section{Introduction}

Continuous opioid infusions (COI) provide fast and effective pain/dyspnoea control in palliative patients. Singapore National Guidelines for Palliative Care (2015) recommend a turnaround time of $<1$ hour for urgent orders. Our team observed that administration of COI was sometimes delayed, which can lead to poor patients' experiences and outcomes. Previous baseline data collected for the COI turnaround time is 101 minutes. A quality improvement project was thus undertaken to achieve timely symptom control for patients needing COI.

\section{$\underline{\text { Aim }}$}

To achieve a turnaround time of $<60$ minutes in $100 \%$ of patients prescribed COI for moderate to severe pain/dyspnoea in one of the medicalsurgical wards of Singapore General Hospital (SGH).

\section{Methodology}

Baseline data was collected from April-May 17. A total of 21 cases was started on COI, of which $57.14 \%$ had moderate to severe symptoms, out of these patients, only $33.33 \%$ had COI set up within 60 minutes. Common causes of delay at each stage of the process were delineated, and brainstorming of possible interventions was done by the study team consisting of a doctor, palliative care nurses, ward nurses and a pharmacist. As this is a quality improvement project, Plan-DoStudy-Act (PDSA) cycles were used to test the effectiveness of each intervention.

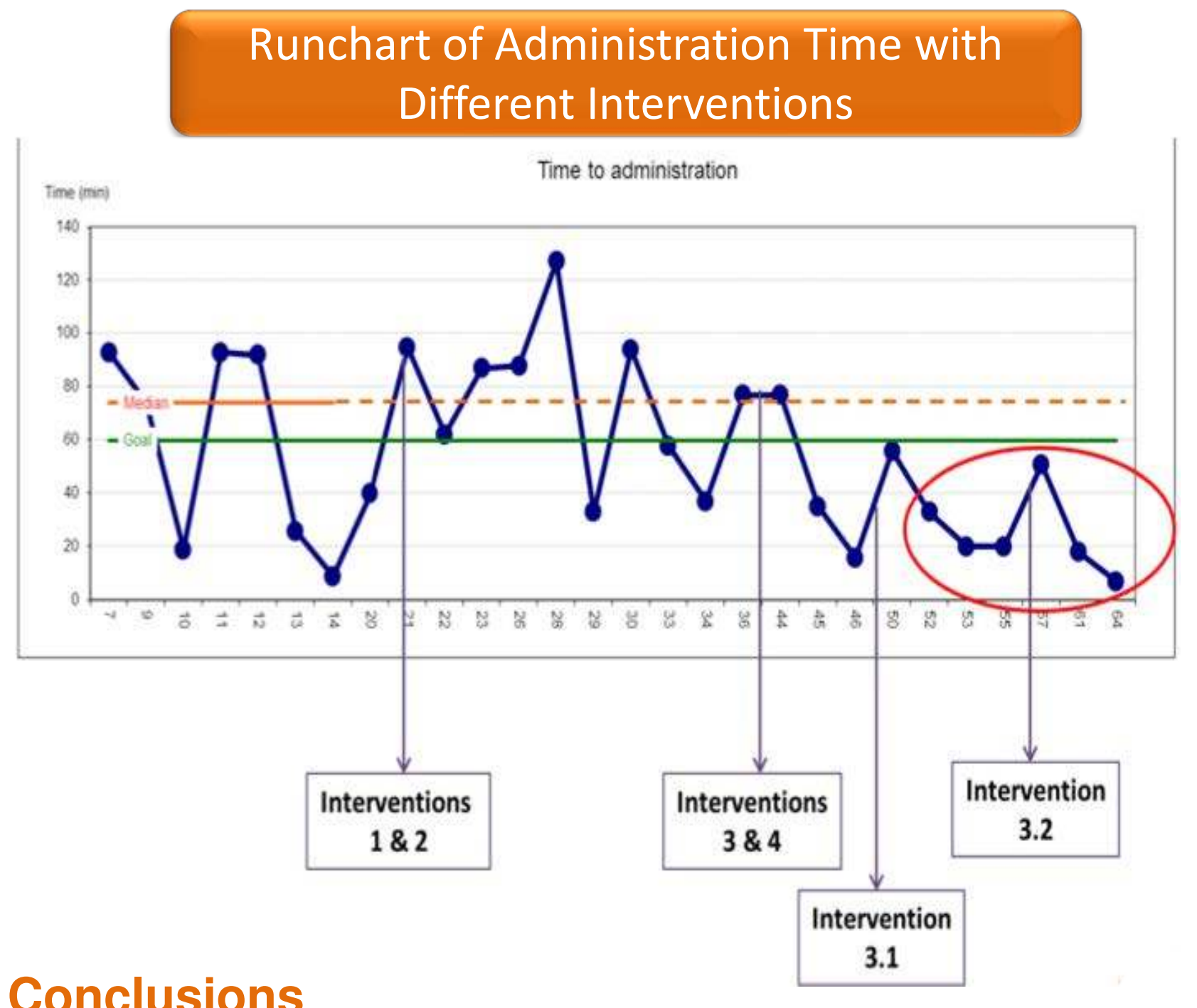

Workflow for Newly Initiated

Continuous Opioid infusion

Dr orders in e-system \& to inform nurse-in-charge for patients newly

initiated on subcutaneous/intravenous continuous Opioid Infusions

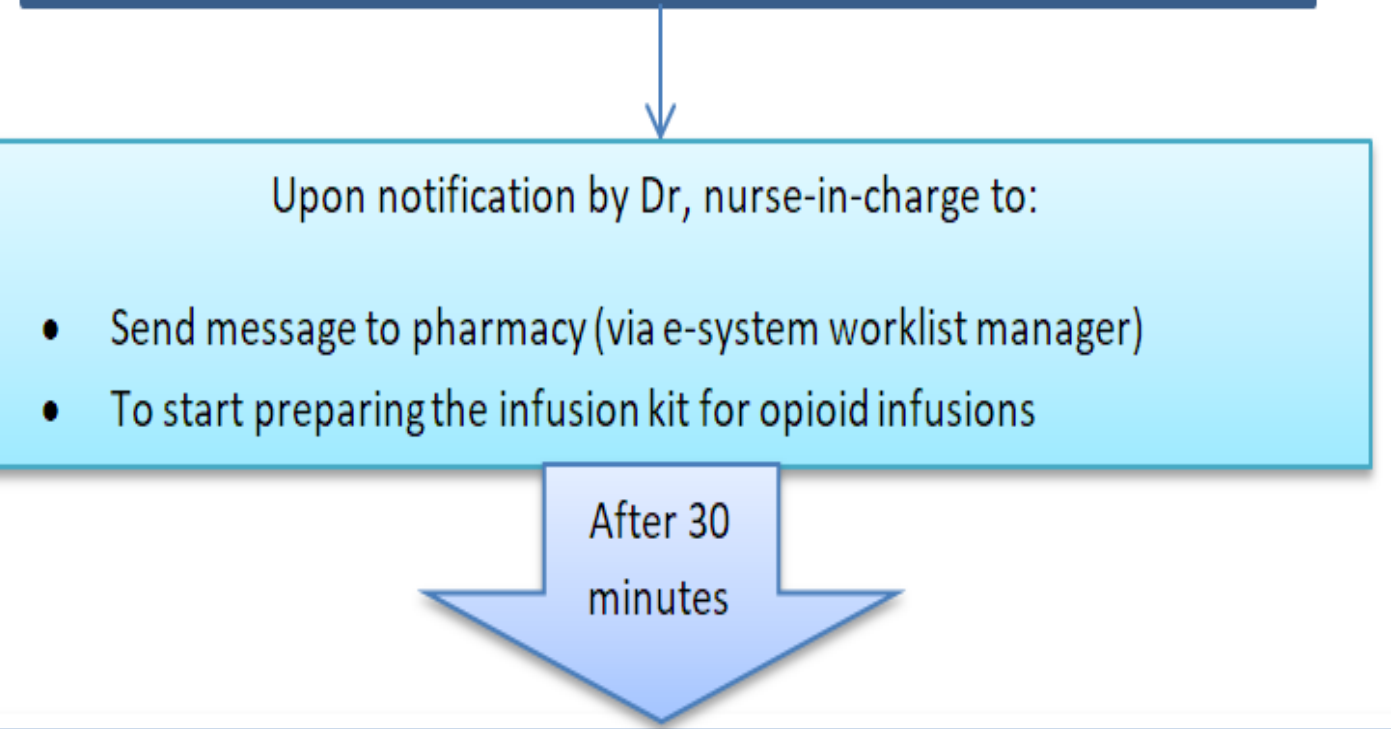

Nurse-in-charge to check if opioid infusion have been verified by pharmacies
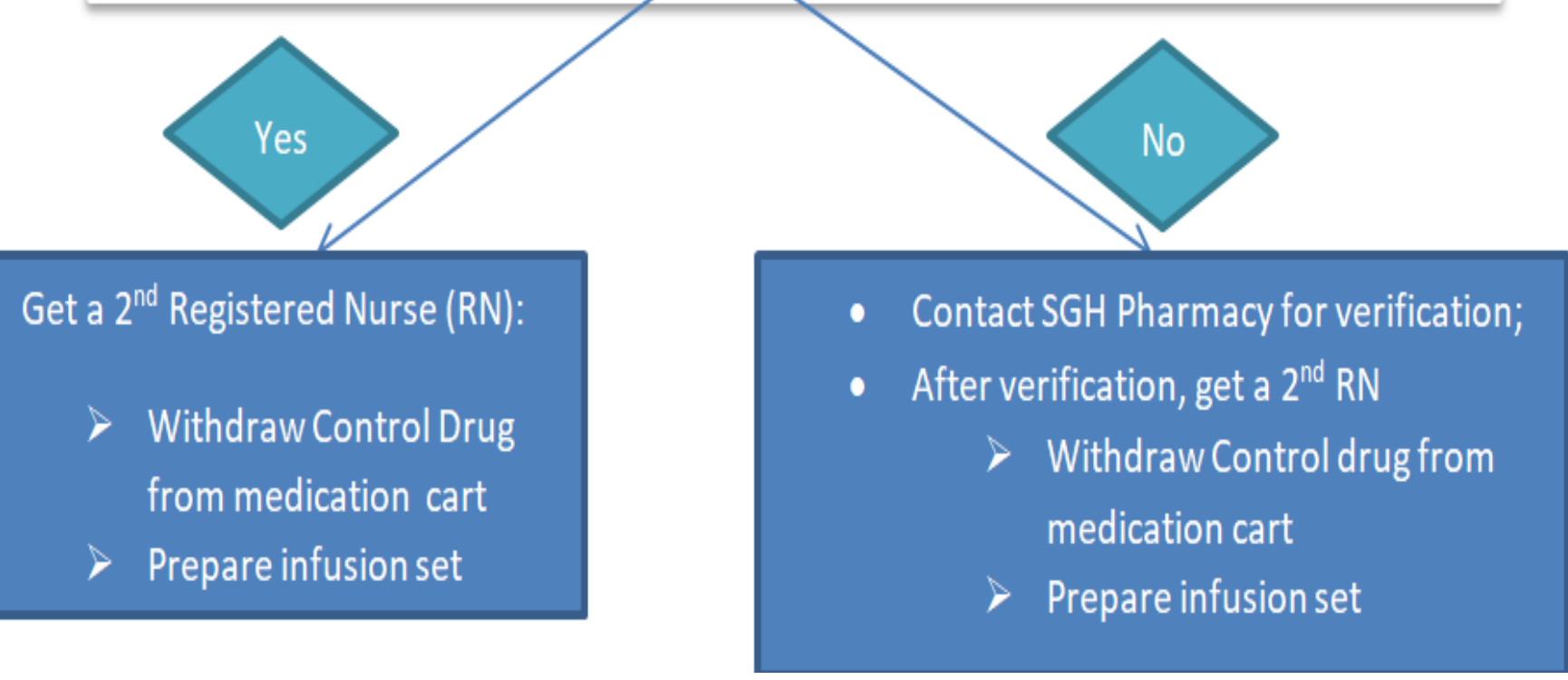

Results

A total of 4 interventions targeting nursing, pharmacy and medical workflows were tested since 25/07/17. The last intervention (3.2) which included a standardized nursing workflow and providing reminders for nurses via flowcharts placed at medication carts resulted in improvements in time taken for administration of COls. $100 \%$ of patients had COls started within 60 minutes from 28/9/17 till 15/1/18. Tremendous improvement was showed with time taken for verification of opioids orders and administration: COI turnaround time of $<1$ hour was achieved with a median of 20 minutes to administer $\mathrm{COI}$ as shown in the Runchart.

Having a standardized workflow and reminders for nurses at highly visible areas such as medication carts displayed effectiveness in reducing COI turnaround time in patients with moderate to severe pain/dyspnoea. 